\title{
Variations in modifications of sugar residues in hamster zona pellucida after in vivo fertilization and in vitro egg activation
}

\author{
M. El-Mestrah and F. W. K. Kan* \\ Department of Anatomy and Cell Biology, Faculty of Health Sciences, \\ Queen's University, Kingston, Ontario K7L 3N6, Canada
}

Lectins were used as probes in conjunction with quantitative analysis to investigate the distribution of different carbohydrate residues in hamster zona pellucida and their possible modification patterns after in vivo fertilization and in vitro egg activation. Several lectins including HPA, WGA, RCA-I, PNA, DSA, BSAIB ${ }_{4}$ DBA, AAA and MAA were used to label the zona pellucida of both unfertilized and fertilized eggs. With the exception of PNA and BSAIB ${ }_{4}$, the same lectins were also used to label the zona pellucida of oocytes activated in vitro. A multicomparison quantitative analysis of the density of labelling in the inner and outer regions of the zona pellucida before and after fertilization in vivo, as well as after in vitro egg activation, was performed. Of all the lectins studied, preferential localization of labelling by RCA-I and DSA to the inner zona pellucida of unfertilized eggs was observed. After in vivo fertilization, there was an increase in labelling in the inner region of the zona pellucida when thin sections of fertilized oocytes were incubated with HPA, BSAIB ${ }_{4}$ and AAA. Although increased labelling by RCA-I was observed, a significant decrease in labelling intensity was obtained with WGA and the sequence Neu-WGA in both the inner and outer zona pellucida of fertilized oocytes. A significant increase in the density of labelling with WGA was also observed after digestion with neuraminidase. In parallel, when hamster oocytes activated in vitro were compared with those fertilized in vivo, a difference in lectin-gold labelling was observed in both the inner and outer region of the zona pellucida. Labelling with HPA, WGA, DSA and MAA increased significantly in both the inner and outer regions of the zona pellucida, whereas labelling by DBA significantly decreased in the inner portion of the zona pellucida. After neuraminidase treatment, a significant increase in labelling density was observed when thin sections of in vitro-activated oocytes were incubated with WGA. These results demonstrate: (i) the post-fertilization modifications of major saccharidic determinants that may play a role in the sperm-egg interaction process of fertilization in vivo; and (ii) that the modified properties of zonae pellucidae of fertilized and in vitro-activated eggs resulting from the action of hydrolytic enzymes, as well as glycoproteins released through exocytosis of cortical granules, are not identical.

\section{Introduction}

The zona pellucida is an amorphous layer of glycoproteins that surrounds mammalian oocytes. It plays a critical role during mammalian oogenesis, fertilization and preimplantation development. The composition of the zona pellucida has been characterized in several mammalian species and the zona pellucida matrix comprises mainly three major glycoproteins (ZP1, ZP2 and ZP3) that show extreme molecular mass and charge heterogeneity as a result of extensive post-translational modifications including glycosylation and sulphation (Wassarman, 1988a; Moller et al., 1990; Dunbar et al., 1994). Both the initial sperm binding activity and acrosome inducing activity have been ascribed to a class of $O$-linked oligosaccharides that are linked covalently to ZP3 (Florman and Wassarman, 1985; Bleil and Wassarman, 1988; Litscher et al., 1995). Another major

*Correspondence

Email:kanfwk@post.queensu.ca glycoprotein, ZP2, is probably responsible for the secondary binding of the acrosome-reacted spermatozoa and its post-fertilization modification may play a role in the block to polyspermy (Bleil et al., 1988; Wassarman, 1988a). ZP1, a homodimer in mice, may serve as a cross-linker of copolymers of ZP2 and ZP3 and, thus, adds structural integrity to the zona pellucida to facilitate the protection of the embryo as it passes down the oviduct (Wassarman, 1988b; Rankin et al., 1999). However, results from other species (rabbits, pigs) indicate that there may be a role for ZP1 homologues in sperm binding (Yonezawa et al., 1995; Prasad et al., 1996). On fusion of a spermatozoon with the egg plasma membrane, a series of events in the egg referred to as 'egg activation' is initiated. During activation, a transient increase in intracellular $\mathrm{Ca}^{2+}$ concentration evokes exocytosis of cortical granule exudate into the perivitelline space, thereby altering the biological properties of the zona pellucida glycoproteins (zona reaction) and establishing a block to polyspermy (Ducibella, 1991). The nature of the 
Table 1. Lectins used for identification of sugar residues in the zona pellucida of unfertilized, fertilized and in vitro-activated hamster eggs

\begin{tabular}{|c|c|c|c|}
\hline Source of lectin & Acronym & Carbohydrate binding specificity & Inhibitory sugar \\
\hline Helix pomatia (Roman snail) & HPA & Terminal $\alpha$ - and $\beta$-linked GalNAc & D-GalNAC \\
\hline Dolichos biflorus (horse gram) & DBA & Terminal $\alpha$-linked GalNAc & D-GalNAc \\
\hline Triticum vulgaris (wheatgerm) & WGA & Terminal non-reducing GlcNAc or Neu5Ac & D-GlcNAc and Neu5Ac \\
\hline Galanthus nivalis (snowdrop bulb) & GNA & $\begin{array}{l}\text { Terminal Man } \alpha 1,3 \text { in high mannose } N \text {-linked } \\
\text { oligosaccharides }\end{array}$ & Methyl- $\alpha$-mannose \\
\hline Ricinus communis (castor bean) & RCA-I & Terminal Gal $\beta 1,4$ GlcNAc disaccharides & D-Galactose \\
\hline Arachis hypogea (peanut) & PNA & Terminal Gal $\beta 1,3$ GalNAc disaccharides & D-Galactose \\
\hline Datura stramonium (Jimson weed) & DSA & $\begin{array}{l}\text { Bi-, tri- and tetra-antennary sugar chains with at } \\
\text { least one } N \text {-acetyllactosamine present }\end{array}$ & N-Acetyllactosamine \\
\hline Bandaeiraea simplicifolia (seeds) & $\mathrm{BSAIB}_{4}$ & Terminal $\alpha$-linked galactose sugar residues & Methyl- $\alpha$-galactose \\
\hline Ulex europaeus (asparagus pea) & UEA-I & Terminal $\alpha$-L-fucose & L-Fucose \\
\hline Lotus tetragonolobus (asparagus pea) & LTA & $\begin{array}{l}\text { Type } 2 \text { chains with fucose on C- } 2 \text { of the galactose } \\
\text { of Gal } \alpha 1,4 \text { Glc NAc }\end{array}$ & L-Fucose \\
\hline Aleuria aurantia (fresh water snail) & AAA & $\begin{array}{l}\text { Complex-type } N \text {-linked oligosaccharides with an } \\
\alpha 1,6 \text { fucosyl residues at the innermost Glc NAc }> \\
\text { terminal } \alpha 1,2 \text { fucose }\end{array}$ & L-Fucose \\
\hline Maackia amureusis (seeds) & MAA & Neu5Ac $\alpha 2,3$ Gal $\beta 1,4$ GIc NAc & $\mathrm{N}$-acetylneuraminyllactose \\
\hline Sambucus nigra (elderberry) & SNA & Neu5Ac $\alpha 2,6 \mathrm{Gal}$ & $\mathrm{N}$-acetylneuraminyllactose \\
\hline
\end{tabular}

GalNAc: N-acetyl-D-galactosamine; GlcNAc: N-acetyl-D-glucosamine; Gal: galactose; Man: mannose; Fuc: fucose; Neu5Ac: N-acetylneuraminic acid.

biochemical alterations occurring in the zona pellucida during fertilization or egg activation is only partially understood. ZP2 undergoes a limited proteolytic cleavage and is converted to a form called ZP2 $\mathrm{f}$ which is associated with the block to polyspermy (Bleil et al., 1981; Moller et al., 1990), whereas ZP3 loses both sperm receptor activity and the ability to induce the acrosome reaction (Bleil and Wassarman, 1988; Moller et al., 1990); however, the migration of ZP3 on SDS-polyacrylamide gels was unchanged after the cortical granule reaction (Miller et al., 1992). Although the role of at least a portion of ZP3 polypeptide backbone has been implicated in induction of the sperm acrosome reaction (Wassarman, 1988a), the above data imply clearly the crucial role of the carbohydrate moiety of ZP3-linked oligosaccharides in such post-fertilization changes. Several monosaccharide residues on ZP3, including $\mathrm{N}$-acetylglucosamine, $\mathrm{N}$-acetylgalactosamine, mannose, fucose, galactose and sialic acid have been implicated as the complementary sperm receptors, mediating the primary binding between the spermatozoon and the zona pellucida (Bleil and Wassarman, 1988; Miller et al., 1992; Litscher et al., 1995; Thaler and Cardullo, 1996; Benoff, 1997; Tulsiani et al., 1997; Johnston et al., 1998). However, the exact oligosaccharide sequence or sequences that mediate initial sperm-egg binding is not known. In the present study, high resolution lectin-gold cytochemistry was used with electron microscopy to characterize the distribution of various terminal oligosaccharide sugar residues of zona pellucida glycoproteins in both unfertilized and fertilized hamster eggs, as well as in hamster eggs activated in vitro. The multicomparison quantitative analysis of the density of labelling obtained with various lectin probes provides a means to evaluate the anticipated modifications of the different sugar residues in the bilaminar zona matrix after fertilization in vivo and presents further insights into the role of cortical granule exudate in bringing about these modifications.

\section{Materials and Methods}

\section{Reagents}

Calcium ionophore A23187, polyethylene glycol (molecular mass 20 000), eCG, hCG, sodium citrate, tetrachloroauric acid $\left(\mathrm{HauCl}_{4} \cdot 2 \mathrm{H}_{2} \mathrm{O}\right)$, hyaluronidase (type $\mathrm{VI}$, from bovine testis), neuraminidase (type $\mathrm{V}$, from Clostridium perfringens), BSA and galactose oxidase (from Dactylium dendroides) were purchased from Sigma (St Louis, MO). Colloidal gold-conjugated lectins (DSA and DBA), unlabelled lectins (HPA, RCA-I, LTA, UEA-I, BSAIB 4 and WGA), ovomucoid, D-galactose, L-fucose, N-acetylglucosamine, $\mathrm{N}$-acetyl-D-galactosamine, $\mathrm{N}$-acetylneuraminic acid, $N$-acetylneuraminyllactose, methyl- $\alpha$-D-mannopyrannoside, methyl- $\alpha$-galactopyrannoside and $N$-acetyllactosamine were obtained from EY laboratories, Inc. (San Mateo, CA). Digoxigenin (DIG)-labelled lectins (AAA, MAA, SNA, GNA and PNA) and mouse monoclonal IgG anti-DIG antibodies were purchased from Roche Diagnostics (Quebec). Goat anti-mouse IgG + IgM-gold complex (15 nm) was purchased from Cedarlane Research Ltd (Ontario). The taxonomic names and specificities of the lectins used in the present study are shown (Table 1).

\section{Collection and preparation of oocytes and fertilized eggs for lectin cytochemistry}

Twenty sexually mature female golden hamsters (Mesocricetus auratus), aged 7-8 weeks, were purchased 
from Charles River Laboratories (St Constant). The hamsters were tested for the typical vaginal secretion that is found in abundance at metoestrus to obtain oviductal oocytes. The oestrous cycles of the hamsters were observed for 2-3 consecutive weeks to ascertain their regularity. Four animals were superovulated by i.p. injection of 25 iu eCG the day before the expected oestrus followed by another injection of 25 iu hCG 48 h later (Flemming and Yanagimachi, 1980). The animals were killed by cervical dislocation $17 \mathrm{~h}$ after injection with hCG to obtain cumulus masses (made up of unfertilized oocytes and the associated cumulus cells). The ventral abdominal wall of each animal was immediately cut open and the oviducts were excized. The ampullary portion of the oviduct was identified and torn open under a dissecting microscope using fine tweezers, and the cumulus masses were collected in PBS, pH 7.4, washed briefly in PBS and fixed by immersion at room temperature for $2 \mathrm{~h}$ in $2.5 \%(\mathrm{v} / \mathrm{v})$ glutaraldehyde in $0.1 \mathrm{~mol}$ cacodylate buffer $\mathrm{I}^{-1}$, $\mathrm{pH}$ 7.4. For collection of fertilized eggs, four female hamsters were placed with fertile males on the evening before oestrus. Fertilized eggs were collected from the oviducts of females killed by cervical dislocation on day 1 after the animals were mated. The eggs were fixed at room temperature by immersion in $2.5 \%(\mathrm{v} / \mathrm{v})$ glutaraldehyde in 0.1 mol cacodylate buffer $\mathrm{I}^{-1}(\mathrm{pH} 7.4)$ for $2 \mathrm{~h}$. At the end of fixation, the cumulus masses and fertilized eggs were washed three times in 0.1 mol cacodylate buffer $\mathrm{I}^{-1}$ and left overnight at $4^{\circ} \mathrm{C}$ in the same buffer. For post-embedding labelling, fertilized eggs were embedded in a $3 \%(\mathrm{w} / \mathrm{v})$ gelatin solution, trimmed into small cubes, dehydrated in a series of graded ethanols, infiltrated and embedded in LR White (Electron Microscopic Sciences, Fort Washington, PA) according to routine procedures. The cumulus masses were processed in the same way without trimming. Areas of interest were first located in sections of $1 \mu \mathrm{m}$ thickness of LR White-embedded specimens by light microscopy. Thin sections of cumulus masses and fertilized eggs were cut with a diamond knife on an LKB ultramicrotome and mounted on formvar-carbon-coated nickel grids.

\section{lonophore activation of eggs}

Cumulus masses were collected from the oviductal ampullae of 12 superovulated female hamsters prepared as described above. Cumulus cells were removed by digestion for $5 \mathrm{~min}$ in $1 \mathrm{mg}$ testicular hyaluronidase $\mathrm{ml}^{-1}$. Cumulusfree eggs were washed by consecutive transfer through at least six droplets of dmKRBT buffer $\left(120 \mathrm{mmol} \mathrm{NaCl} \mathrm{I}^{-1}\right.$, $2 \mathrm{mmol} \mathrm{KCl} \mathrm{I-1}, 2 \mathrm{mmol} \mathrm{CaCl}_{2}{ }^{-1}, 10 \mathrm{mmol} \mathrm{NaHCO}_{3}{ }^{-1}$, $1.2 \mathrm{mmol} \mathrm{MgSO}_{4} \mathrm{I}^{-1}, 5.6 \mathrm{mmol}$ glucose $\mathrm{I}^{-1}, 1.1 \mathrm{mmol}$ sodium pyruvate $\mathrm{I}^{-1}, 25 \mathrm{mmol}$ TAPSO (3-[N-Tris (hydroxymethyl) methylamino]-2-hydroxy propanesulphonic acid) $\mathrm{I}^{-1}, 18.5 \mathrm{mmol}$ sucrose $\mathrm{I}^{-1}, 6 \mathrm{mg} \mathrm{BSA} \mathrm{ml}^{-1}$, pH 7.3; Neill and Olds-Clarke, 1988; Miller et al., 1993) covered with mineral oil to ensure the removal of hyaluronidase and contaminating glycosidases. The eggs ( $n=100)$ were activated parthenogenetically within $1 \mathrm{~h}$ of collection to avoid the deleterious effects of egg ageing, by incubation in medium containing $10 \mu \mathrm{mol} \mathrm{I-1}$ (final concentration) of the calcium ionophore A23187 in dmKRBT, under mineral oil, for $30 \mathrm{~min}$ at $37^{\circ} \mathrm{C}$ in a chamber acclimatized with 5\% $\mathrm{CO}_{2}$ (Cherr et al., 1988; Ducibella et al., 1988). Stock solution of calcium ionophore A23187 was prepared at $10 \mathrm{mmol} \mathrm{I}^{-1}$ in dimethylsulphoxide (DMSO) and kept at $4^{\circ} \mathrm{C}$ until used. For controls, a total of 50 eggs was placed in a medium containing dmKRBT only and incubated under the same conditions. Both ionophoreactivated and control eggs were washed extensively at least six times (5 min each) by consecutive transfer through droplets of dmKRBT covered with mineral oil. The eggs were finally transferred to beem capsules (CANEMCO, St Laurent) and fixed for $2 \mathrm{~h}$ in $2.5 \%(\mathrm{v} / \mathrm{v})$ glutaraldehyde in $0.1 \mathrm{~mol}$ cacodylate buffer $\mathrm{I}^{-1}$ at room temperature. After fixation, both control and ionophore-activated eggs were washed three times in 0.1 mol cacodylate buffer $\left.\right|^{-1}$. For post-embedding lectin cytochemistry, activated and control eggs were processed further in the same way as unfertilized and fertilized oocytes.

\section{Preparation of colloidal gold, lectin-gold and glycoprotein-gold complexes}

Colloidal gold solutions with a mean particle diameter of $15 \mathrm{~nm}$ were prepared by the sodium citrate method as described by Frens (1973). Ovomucoid-gold and lectin (HPA, RCA-I, LTA and UEA-I)-gold complexes were prepared as described by Geoghegan and Ackerman (1977). The minimal amounts of lectins or protein needed for stabilization of colloidal gold and the optimal $\mathrm{pH}$ were estimated by the method of Geoghegan and Ackerman (1977).

\section{Cytochemical labelling}

Colloidal gold was used as a marker for lectin cytochemistry carried out at the ultrastructural level. Cytochemical labelling was performed by the one-step (Roth, 1983; Benhamou, 1986), two-step (Geoghegan and Ackerman, 1977; Benhamou, 1986) or three-step (MartínezMenárguez et al., 1992) methods. The one-step method was used for HPA, RCA-I, UEA-I, LTA, DBA and BSAIB 4 . Ultrathin tissue sections were first incubated on a drop of $0.5 \%(\mathrm{v} / \mathrm{v}) \mathrm{BSA}$ in $0.01 \mathrm{~mol} \mathrm{PBS} \mathrm{l}^{-1}(\mathrm{pH} \mathrm{7.4)}$ for $5 \mathrm{~min}$ and transferred on to a drop of the lectin-gold complex for $1 \mathrm{~h}$. The sections were washed with PBS followed by bidistilled water and dried on a filter paper. For WGA, the two-step method was applied. In brief, tissue sections were floated for 5-10 min on a droplet of $0.5 \%(\mathrm{v} / \mathrm{v})$ BSA in PBS and transferred to a drop of unlabelled WGA diluted in PBS for $1 \mathrm{~h}$. After PBS washing, the sections were floated on a droplet of ovomucoid-gold complex for $30 \mathrm{~min}$ followed by rinsing with PBS and double-distilled water. For lectin-DIG labelling (AAA, MAA, PNA, GNA and SNA), a three-step method was used as described by Martínez-Menárguez et al. (1992) and Avilés et al. (1996). 
The above incubations were all performed at room temperature. The sections were counterstained with uranyl acetate and lead citrate before examination on a Hitachi 7000 electron microscope operated at $75 \mathrm{kV}$.

\section{Cytochemical controls}

The labelling specificities of the lectins were assessed by the following controls (incubations were all carried out at room temperature unless indicated otherwise): (i) substitution of conjugated or unconjugated lectins by the corresponding buffer; (ii) reincubation of the lectins with the corresponding hapten-sugar inhibitor at concentrations ranging from $0.1 \mathrm{~mol} \mathrm{I}^{-1}$ to $0.2 \mathrm{~mol} \mathrm{I}^{-1}$ : $\mathrm{N}$-acetyl-Dgalactosamine (GalNAc) (for HPA and DBA), D-galactose (for RCA-I and PNA), D-N-acetyl-D-glucosamine (GlcNAc) and/or N-acetylneuraminic acid (Neu5Ac) (for WGA), methyl- $\alpha$-mannose (for GNA), $N$-acetyllactosamine (for DSA), L-fucose (for AAA, UEA-I and LTA), methyl- $\alpha$ galactose (for $\mathrm{BSAIB}_{4}$ ) and $\mathrm{N}$-acetylneuraminyllactose (for MAA and SNA); (iii) preincubation of tissue sections with neuraminidase (1 iu $\mathrm{ml}^{-1}$ ) for WGA and MAA, and with galactose oxidase for HPA and PNA.

\section{Enzymatic treatment and controls}

WGA has an affinity for GlcNAc and Neu5Ac residues. For neuraminidase treatment, tissue sections were digested with 1 iu neuraminidase in acetate buffer $\mathrm{ml}^{-1}, \mathrm{pH} 5.0$, for $3 \mathrm{~h}$ at $37^{\circ} \mathrm{C}$ before labelling with the lectin to remove Neu5Ac residues. For galactose oxidase treatment, ultrathin sections were incubated with 50 iu galactose oxidase in PBS $\mathrm{ml}^{-1}, \mathrm{pH} 7.4$, for $24 \mathrm{~h}$ at $37^{\circ} \mathrm{C}$ in a moist chamber before labelling with the corresponding lectin.

Controls for both neuraminidase and galactose oxidase treatments were performed by substitution of the enzymes with their corresponding buffers.

\section{Quantitative analysis}

Quantitative evaluation of the lectin-gold labelling was performed as described by Avilés et al. (2000a). For tissue sections that showed positive reactivity to lectins, the labelling densities over the zona pellucida of both unfertilized and fertilized, as well as of in vitro-activated, oocytes were evaluated on positive electron micrographs enlarged to $\times 22500$. For each of the lectins under study, the density of labelling, evaluated as the number of gold particles per $\mu \mathrm{m}$ square surface area, was determined by use of a Carl Zeiss MOP-3 modular system equipped for quantitative digital image analysis.

The heterogeneous nature of the hamster zona pellucida has been revealed by lectin cytochemistry (Yanagimachi and Nicolson, 1976; Ahuja and Bolwell, 1983). More recently, the hamster zona pellucida has been shown to present a multilaminar structure by non-invasive polarized light microscopy and digital image processing (Keefe et al., 1997). As such, the hamster zona pellucida appeared to consist of two birefringent layers separated by an anisotrophic layer. In the present study, for the purposes of quantitative analysis, the zona pellucida was separated into inner and outer zones on photomicrographs by drawing a line separating the zona pellucida into two equal halves concentrically. Electron micrographs were taken at random of ten different regions of the zona pellucida of unfertilized and fertilized oocytes ( $n=4$ animals in each case, 1-2 oocytes per animal), as well as of activated oocytes (five randomly selected oocytes in total). Fifteen to twenty fields (3-5 $\mu \mathrm{m}^{2}$ each) of both the outer and inner zona pellucida from each egg were used for the quantitative evaluation (Table 2). Labelling densities were compared using one-way analysis of variance with $P<0.001$ and a Student-NewmanKeuls method with $P<0.05$ performed with a SigmaStat program (Jandel, San Rafael, CA), respectively. A multicomparison statistical analysis of the differences in the mean labelling densities for the lectin-gold labelling was undertaken for: (i) lectin labelling in the outer and inner zona pellucida within each of the three experimental groups (unfertilized, fertilized and in vitro-activated oocytes; (ii) lectin labelling in the inner and outer zona pellucida of oocytes before and after fertilization; (iii) lectin labelling in the inner and outer zona pellucida of fertilized and in vitroactivated oocytes; and (iv) lectin labelling before and after neuraminidase digestion for each of the three experimental groups.

In the present study, the number of gold particles present in control sections of unfertilized, fertilized and in vitroactivated oocytes incubated with various lectins in the presence of their corresponding blocking sugars was negligible and, therefore, was not included in the quantitative analysis.

\section{Results}

Distribution of specific sugar residues of oligosaccharide side chains in the oocyte proper and in the zona pellucida of unfertilized, fertilized and in vitro-activated oocytes

Electron microscope analysis of thin sections of hamster ovulated oocytes (unfertilized oocytes) labelled with HPA (Fig. 1a), WGA (Fig. 2a), PNA, BSAIB 4 , DBA, AAA and MAA, including the sequence Neu-WGA, presented a homogeneous distribution throughout the zona matrix. However, labelling of ovulated eggs with DSA (Fig. 3a) and RCA-I revealed an asymmetric distribution of the corresponding binding sites in the zona pellucida. The density of labelling varied among different lectins ranging from moderate to intense. In the oocyte proper, various structures, including the Golgi apparatus and its associated vesicles, and the vesicular aggregates (made up of several large vesicles embedded in a mass of much smaller and smooth vesicles) displayed a moderate to strong labelling by gold particles. Below the oolemma, cortical granules reacted strongly to labelling by AAA, RCA-I and PNA, but 
Table 2. Labelling densities in the zona pellucida of unfertilized, fertilized and in vitro-activated hamster eggs after incubation with different lectins

\begin{tabular}{|c|c|c|c|c|}
\hline Lectins & Zona pellucida & Unfertilized eggs & Fertilized eggs & Activated eggs \\
\hline \multirow[t]{2}{*}{ HPA } & Inner & $93.66 \pm 5.33$ & $135.54 \pm 8.40^{\mathrm{ab}}$ & $275.05 \pm 8.61^{\mathrm{ac}}$ \\
\hline & Outer & $118.55 \pm 5.42$ & $92.87 \pm 7.71$ & $169.90 \pm 8.83^{c}$ \\
\hline \multirow[t]{2}{*}{ DBA } & Inner & $8.66 \pm 1.31$ & $12.48 \pm 1.27$ & $6.58 \pm 0.98^{c}$ \\
\hline & Outer & $10.45 \pm 1.70$ & $8.06 \pm 1.29$ & $5.23 \pm 0.45$ \\
\hline \multirow{2}{*}{ WGA } & Inner & $79.09 \pm 4.05$ & $47.79 \pm 3.26^{b}$ & $66.91 \pm 3.51^{\mathrm{c}}$ \\
\hline & Outer & $68.32 \pm 4.79$ & $47.03 \pm 2.30^{b}$ & $58.69 \pm 5.79^{c}$ \\
\hline \multirow[t]{2}{*}{ Neu-WGA } & Inner & $97.11 \pm 5.08^{d}$ & $72.75 \pm 4.91^{\mathrm{bd}}$ & $92.68 \pm 4.50^{d}$ \\
\hline & Outer & $92.57 \pm 7.93^{d}$ & $65.05 \pm 4.32^{\mathrm{bd}}$ & $84.59 \pm 9.04^{d}$ \\
\hline \multirow[t]{2}{*}{ RCA-I } & Inner & $122.91 \pm 4.77^{a}$ & $177.53 \pm 4.32^{\mathrm{ab}}$ & $164.31 \pm 12.87^{a}$ \\
\hline & Outer & $102.67 \pm 2.88$ & $145.58 \pm 3.20^{\mathrm{b}}$ & $128.56 \pm 7.15$ \\
\hline \multirow[t]{2}{*}{ PNA } & Inner & $17.71 \pm 1.31$ & $15.65 \pm 2.11$ & na \\
\hline & Outer & $12.09 \pm 1.39$ & $12.70 \pm 1.03$ & na \\
\hline \multirow[t]{2}{*}{ DSA } & Inner & $37.05 \pm 2.31^{\mathrm{a}}$ & $28.66 \pm 2.10^{\mathrm{a}}$ & $75.47 \pm 7.74^{\mathrm{ac}}$ \\
\hline & Outer & $8.13 \pm 0.85$ & $7.95 \pm 0.64$ & $22.84 \pm 2.30^{\mathrm{c}}$ \\
\hline \multirow[t]{2}{*}{$\mathrm{BSAIB}_{4}$} & Inner & $20.71 \pm 0.95$ & $34.85 \pm 2.61^{\mathrm{ab}}$ & na \\
\hline & Outer & $18.46 \pm 1.08$ & $21.38 \pm 1.46$ & na \\
\hline \multirow[t]{2}{*}{ AAA } & Inner & $77.92 \pm 3.62$ & $99.78 \pm 3.57^{b}$ & $91.16 \pm 4.60$ \\
\hline & Outer & $80.36 \pm 3.60$ & $84.14 \pm 1.86$ & $83.32 \pm 2.48$ \\
\hline \multirow[t]{2}{*}{ MAA } & Inner & $25.58 \pm 1.92$ & $27.26 \pm 2.30$ & $76.45 \pm 3.67^{\mathrm{ac}}$ \\
\hline & Outer & $19.46 \pm 1.18$ & $18.02 \pm 1.02$ & $87.23 \pm 6.03^{c}$ \\
\hline
\end{tabular}

Values are gold particles per $\mu \mathrm{m}^{2}$ (mean \pm SEM). na: not assessed.

asignificant difference between inner and outer zones of the zona pellucida $(P<0.05)$.

bSignificant difference between unfertilized and fertilized eggs $(P<0.05)$.

cSignificant difference between fertilized and activated eggs $(P<0.05)$.

dSignificant difference after neuraminidase treatment $(P<0.05)$.

only weakly to HPA, DBA, BSAIB 4 , WGA and MAA. El-Mestrah and Kan (2001) reported similar lectin labelling results in the cortical granules. Other structures inside the oocyte, such as mitochondria, rough endoplasmic reticulum and stacks of annulate lamellae were not labelled. The perivitelline space, located between the inner boundary of the zona pellucida and the surface of ovulated oocytes, was virtually unlabelled by any of the lectins used. A representative example is shown (Fig. 1a).

Noticeable differences in the lectin binding pattern of some of the lectins used in this study were observed in the zona pellucida of fertilized eggs. Unlike unfertilized oocytes, in which the binding pattern of $\mathrm{HPA}, \mathrm{BSAlB}_{4}$ and AAA was homogeneous in the zona pellucida, fertilized eggs labelled with the same lectins and DSA (Fig. 3b), as well as RCA-I, displayed a heterogeneous distribution of gold particles over the entire zona pellucida (see Table 2 for statistical analysis). However, no significant difference was noted in the distribution of labelling by DBA, PNA and MAA, as well as by Neu-WGA, between the inner and outer regions of the zona pellucida. Tissue sections prepared from activated oocytes displayed a heterogeneous distribution in colloidal gold labelling over the zona pellucida with HPA (Fig.1b), DSA (Fig. 3c), RCA-I and MAA (Table 2). A noticeably higher concentration of gold labelling was detected in the inner zona pellucida with DSA (Fig. 3c) and RCA-I, as well as in both the inner and outer zona pellucida with HPA
(Fig. 1b). When tissue sections of both fertilized and in vitroactivated oocytes were labelled with each of the aforementioned lectins, the pattern of labelling by gold particles over the various cellular organelles in the oocyte proper was similar to that observed in the unfertilized oocytes. Of particular interest was the detection of a specific association of intense labelling over the oolemma and in the perivitelline space of fertilized and in vitro-activated eggs with almost all the lectins used in this study (Figs $1 \mathrm{~b}$ and $3 \mathrm{C}$ ). No labelling was observed with UEA-I, LTA, GNA and SNA.

Control tissue sections of unfertilized, fertilized and in vitro-activated oocytes showed a negative reaction to all lectins examined (see Materials and Methods) in the zona pellucida and over previously labelled subcellular structures, thereby demonstrating the specificity of the labels. However, after neuraminidase treatment there was a noticeable increase in WGA labelling in the zona pellucida, whereas an abolition of MAA labelling was observed over the same structure (see Table 2 for quantitative results).

\section{Comparative quantitative analysis of the density of labelling in the zona pellucida of unfertilized and fertilized oocytes, and in vitro-activated oocytes}

Considering the heterogeneous nature of the zona pellucida matrix, a multicomparison quantitative analysis was undertaken between the inner and outer zona pellucida 

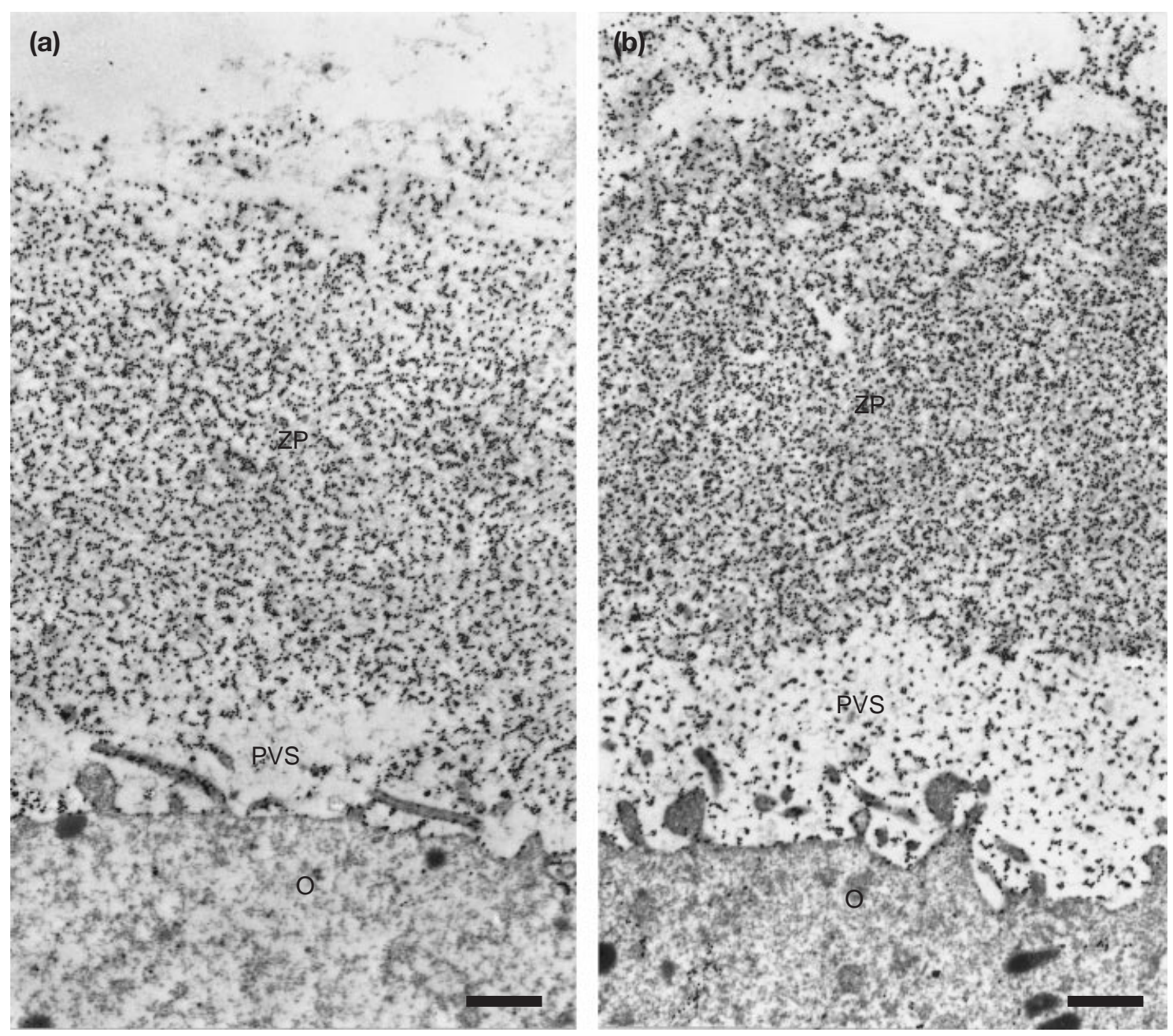

Fig. 1. Helix pomatia agglutinin (HPA)-gold labelling. (a) In the unfertilized hamster oocyte, gold labelling of HPA occupies the entire thickness of the zona pellucida (ZP) with no reactivity observed in the perivitelline space (PVS). (b) An increase in HPA labelling in the inner and outer layers of the zona pellucida is evident after in vitro activation. Note that the perivitelline space of this in vitro-activated egg is heavily labelled with gold particles. O: ooplasm. Scale bars represent (a) 150 and (b) $130 \mu \mathrm{m}$.

within each of the experimental groups under study (unfertilized, fertilized and in vitro-activated oocytes). A similar multicomparison test was also conducted between the inner and outer zones of the zona pellucida of unfertilized and fertilized oocytes, as well as between the inner and outer layer of the zona pellucida of fertilized and activated eggs, to determine whether there was any modification in the distribution of various sugar residues after in vivo fertilization and in vitro activation. The quantitative evaluation revealed significant differences in the distribution of some sugar residues, thereby confirming our electron microscope observations. As such, there was a significant difference in the density of labelling between the inner and outer layers of the zona pellucida with DSA and RCA -I in unfertilized oocytes, as well as with HPA, RCA-I, DSA and $\mathrm{BSAIB}_{4}$ in fertilized oocytes (see Table 2 for quantitative results). After fertilization, the lectin-binding of $\mathrm{HPA}, \mathrm{BSAIB}_{4}$ and $\mathrm{AAA}$ increased significantly in the inner portion of the zona pellucida, whereas that of RCA-I showed a significant increase in both the inner and outer zona pellucida. However, labelling by WGA and the sequence Neu-WGA was reduced significantly in both the inner and outer zona pellucida of fertilized eggs. No significant differences were detected with PNA, DBA, DSA and MAA in both the inner and outer zones of unfertilized and fertilized eggs. In contrast, a significant difference in the density of gold labelling was obtained between the inner and outer zona pellucida when tissue sections of activated oocytes were incubated with HPA, RCA-I, DSA and MAA. Comparative analysis of the density of gold labelling in the inner and outer zona pellucida of fertilized and in vitro-activated oocytes revealed significant variations between the two experimental groups. Labelling by HPA, WGA, DSA and MAA showed a significant increase in both the inner and outer zona pellucida of activated oocytes, whereas DBA labelling showed a significant decrease in the inner layer of 

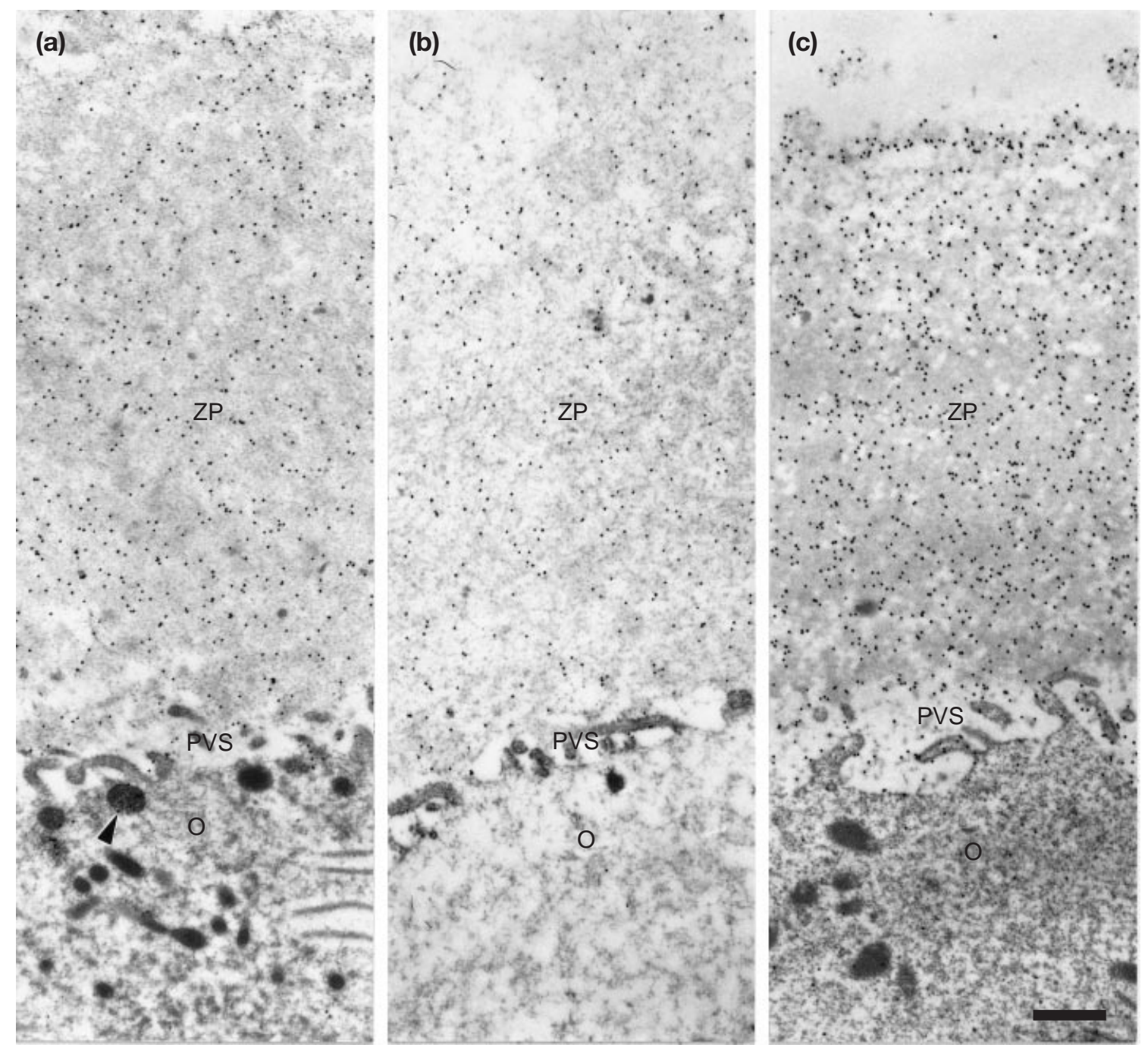

Fig. 2. Triticum vulgaris agglutinin (WGA) labelling followed by incubation with ovomucoid-gold. (a) In the unfertilized hamster oocyte, a homogeneous distribution of gold labelling is observed throughout the zona pellucida (ZP). A cortical granule (arrowhead) is labelled with a few gold particles. (b) After in vivo fertilization, a decrease in WGA labelling over both the inner and outer layer of the zona pellucida is noted. The perivitelline space (PVS) is weakly labelled. (c) After in vitro activation, an increase in WGA labelling is detected in the inner and outer layers of the zona pellucida. Many gold particles are also found in the perivitelline space of this in vitro-activated egg. O: ooplasm. Scale bar represents $150 \mu \mathrm{m}$.

the zona pellucida (see Table 2 for quantitative results). However, no significant differences were found between the inner and outer zonae pellucidae when tissue sections of fertilized and in vitro-activated oocytes were incubated with RCA-I, AAA and the sequence Neu-WGA.

\section{Discussion}

The results obtained in the present study are in agreement with previous studies performed in several other mammals, including rats and mice, using lectin cytochemistry to demonstrate modifications in various carbohydrate residues after fertilization (Raz et al., 1996; Avilés et al., 1997).
Recently, a study using confocal scanning laser microscopy for the detection of lectin-binding glycoconjugates in hamster cortical granules also showed the binding of RCA, PNA, DBA and WGA to the zona pellucida of unfertilized hamster oocytes (Hoodbhoy and Talbot, 2001). However, these authors did not attempt to quantify the density of lectin labelling in the inner and outer regions of the zona pellucida before and after fertilization or after egg activation. Previous studies in several mammalian species demonstrated the occurrence of GalNAc residues as a component of zona pellucida glycoproteins (Shimizu and Ito, 1986; Kimura et al., 1991; Yurewiez et al., 1991; Avilés et al., 1996). Cahova and Draber (1992) showed that pre- 
(a)

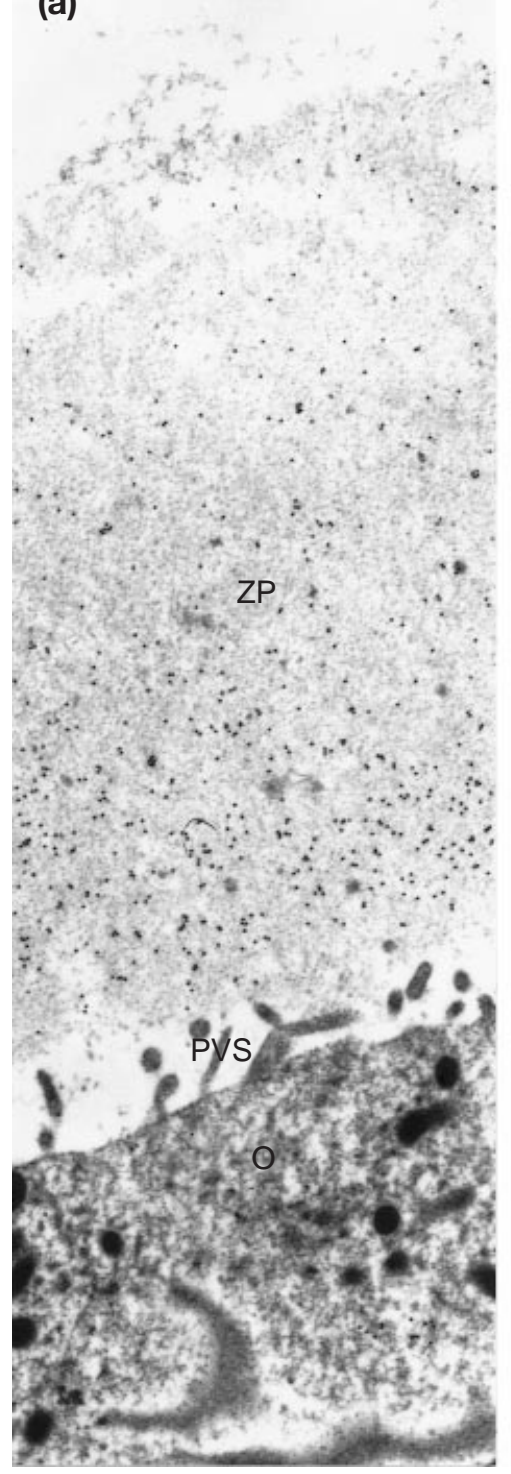

(b)

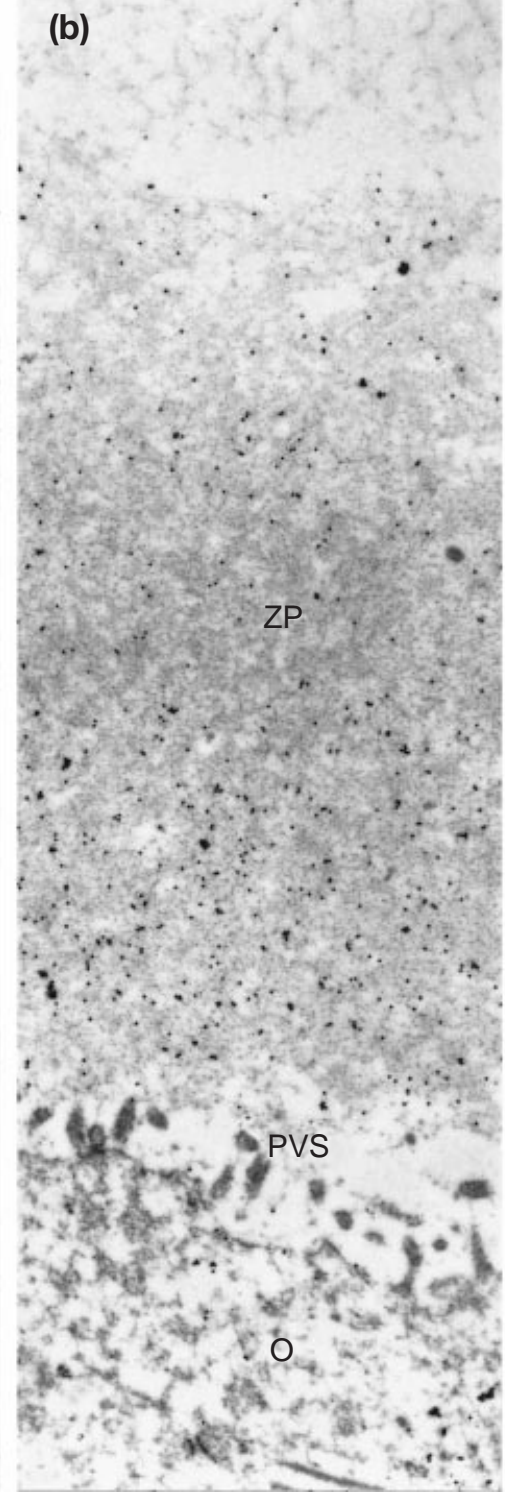

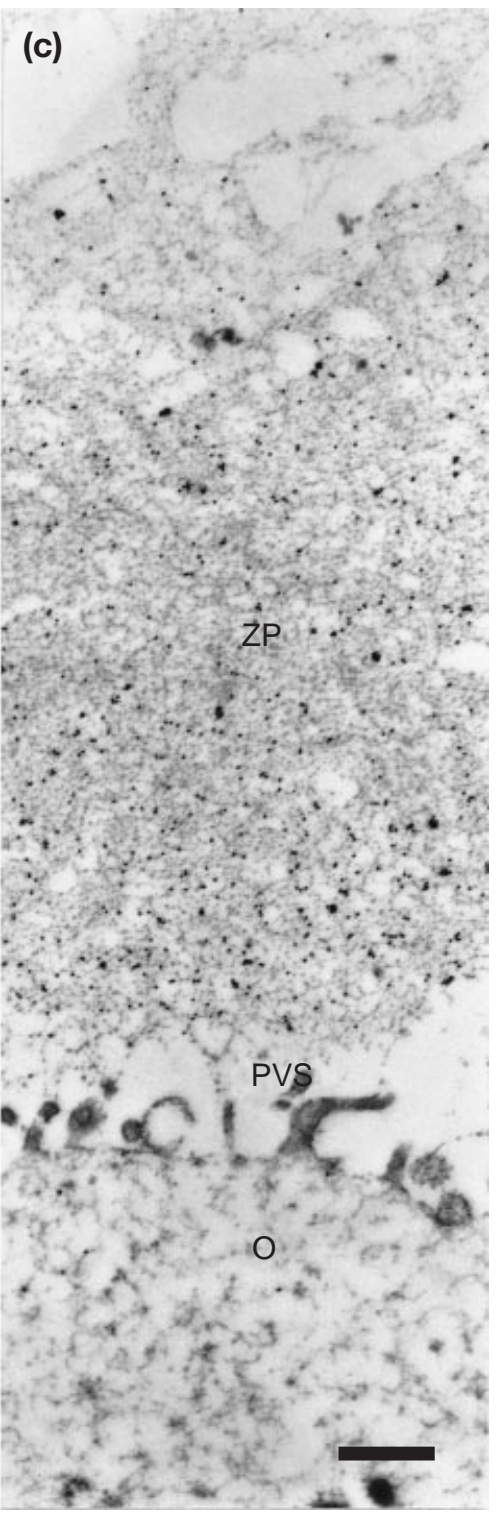

Fig. 3. Datura stramonium (DSA)-gold labelling. (a) An unfertilized hamster oocyte showing preferential distribution of labelling in the inner layer of the zona pellucida (ZP). (b) No apparent change in the distribution of DSA-gold labelling is observed in the inner and outer layers of the zona pellucida of the fertilized oocyte. Labelling is absent in the perivitelline space (PVS) of this fertilized oocyte. (c) An in vitro-activated oocyte showing an increase in DSA labelling in both the inner and outer layers of the zona pellucida. The perivitelline space of this in vitro-activated egg is devoid of any gold labelling. O: ooplasm. Scale bar represents $150 \mu \mathrm{m}$.

incubation of eggs with a monoclonal antibody (TEC-02) directed against the disaccharide GalNAc $\beta 1,4 \mathrm{Gal} \beta 1,4$, which is present in mouse ZP2 and ZP3, inhibits fertilization in vitro; however, the antibody does not interfere with the initial attachment of the spermatozoon to the zona pellucida but inhibits maintenance of the spermatozoonzona pellucida binding (secondary binding). Other studies using the TEC-02 antibody to demonstrate the localization of GalNAc $\beta 1,4 \mathrm{Gal} \beta 1,4$ oligosaccharide sequence to the inner region of unfertilized mouse eggs indicate that the
TEC-02 epitope does not play a role in secondary binding (Avilés et al., 1999). Results from cytochemical studies using HPA lectin (specific for GalNAc sugar residues) in both unfertilized and fertilized mouse eggs indicate that, in mice, non-reducing GalNAc sugar residues are probably not involved in the initial recognition between spermatozoa and the zona pellucida (Avilés et al., 1997). In the present study, the occurrence of non-reducing GalNAc sugar residues over the entire thickness of the zona pellucida in both unfertilized and fertilized hamster oocytes is reported 
using two different lectins: HPA, which preferentially binds non-reducing $\alpha$ - and $\beta$-linked GalNAc residues, and DBA, which predominantly recognizes $\alpha$-GalNAc residues (Piller et al., 1990; Wu and Sugii, 1991). The occurrence of HPA labelling in the outer portion of the zona pellucida indicates a possible role for the non-reducing terminal GalNAc sugar residues in the initial spermatozoon-zona pellucida interaction. This finding is supported further by results from a previous study in hamsters, which showed that spermatozoa mainly recognize the outer region of the zona pellucida (Phillips and Shalgi, 1980).

In the present study, cortical granules were found to react weakly to both HPA and DBA. However, the significant increase in the density of labelling by HPA in the inner region of the zona pellucida and the localization of labelling of both HPA- and DBA- binding receptors at the cell surface of fertilized eggs can be attributed to the incorporation of the enzymatic content of cortical granules into the zona pellucida. Previous studies have implicated enzymes such as proteinases and glycosidases contained in the cortical granules in altering the carbohydrate residues of the zona pellucida glycoproteins, thereby establishing a block to polyspermy (Ducibella, 1991; Miller et al., 1992, 1993). Further studies are required to unravel the anticipated involvement of GalNAc sugar residues in postfertilization hardening of the zona pellucida in addition to their possible roles in sperm-egg recognition and secondary binding.

The presence of both non-reducing $\alpha$ - and $\beta$-galactose residues in both the inner and outer zona pellucida of unfertilized and fertilized eggs was also demonstrated using $\mathrm{BSAIB}_{4}$ and the group RCA-I, PNA and DSA, respectively. However, an increase in $\mathrm{BSAIB}_{4}$ (specific for $\alpha$-galactose) in the inner zona pellucida and an increase in RCA-I (specific for $\beta$-galactose) labelling of both the inner and outer zona pellucida were noted after fertilization. Reactivity to RCA-I revealed the occurrence of Gal $\beta 1,4 \mathrm{Gl}$ N NAc disaccharide throughout the zona pellucida, whereas the presence of the disaccharide Gal $\beta 1,3$ GalNAc was revealed using PNA lectin. Therefore, these results indicate that galactose sugar residues with both $\alpha$-linkage (recognized by BSAIB $_{4}$ ) and $\beta$ linkage (recognized by RCA-I, DSA and PNA) may also be involved in the initial recognition between spermatozoa and the zona pellucida in hamsters. In particular, the results of the present study indicate that $\alpha$ - and $\beta$-galactose sugar determinants may play a role in later stages of sperm penetration. It is possible that, after fertilization, an alteration in the distribution of either $\alpha$-galactose or $\beta$-galactose sugar residues, presumably caused by the release of specific enzymes or other glycoproteins contained in the cortical granules, is necessary as a secondary barrier to prevent additional spermatozoa from entering the fertilized egg. Previous cytochemical studies in mouse and rat ovarian oocytes excluded the role of $\alpha$-galactose in primary spermegg binding, as the latter epitope was confined to the inner portion of the zona pellucida (Avilés et al., 2000b). Therefore, the results of the present study indicate that distribu- tion of $\alpha$-galactose in the zona pellucida varies among rodent species.

GlcNAc sugar residues contained in the zona pellucida of several mammals have been implicated to play a key role in the initiation of fertilization (Berger et al., 1989; Miller et al., 1992, 1993). In mice, it was suggested that the enzyme $\beta$-1,4-galactosyltransferase (GalTase), an integral plasma membrane component of the sperm surface, serves as the sperm receptor by binding to terminal GlcNAc residues on mouse ZP3 (Miller et al., 1992; Shur, 1993; Gong et al., 1995). Removal of ZP3 terminal GlcNAc residues by $N$ acetylglucosaminidase digestion caused a loss of ZP3 sperm receptor activity (Miller et al., 1992). It has been proposed that the enzyme, contained predominantly in the cortical granules of mouse eggs, acts on ZP3 to remove terminal GlcNAc residues, thereby inactivating ZP3 sperm receptor activity and establishing the block to polyspermy (Miller et al., 1993). In the present study, a decrease in labelling with WGA and the sequence Neu-WGA was detected in both the inner and outer portions of the zona pellucida after fertilization. This occurrence of GlcNAc residues in the outer portion of the zona pellucida in both fertilized and unfertilized eggs, and the subsequent decrease in WGA labelling in the outer zona pellucida after fertilization, indicates that GlcNAc is a sugar component that is likely to be involved in sperm-zona pellucida interaction. Our results also demonstrated labelling by Neu-WGA in the cortical granules of unfertilized eggs (results not shown) and the occurrence of a high intensity of labelling in the perivitelline space of fertilized eggs after labelling with WGA and Neu-WGA. Therefore, it is likely that the decrease in the binding of both WGA and Neu-WGA in both the inner and outer zona pellucida of fertilized eggs is caused by the release of specific glucosaminidase, similar to that reported in mice (Miller et al., 1992), into the perivitelline space during the cortical reaction. Further evidence for this contention is provided by results from previous studies that demonstrated structural and functional similarities between hamster and mouse zona pellucida glycoproteins (Moller et al., 1990).

In the present study, the zona pellucida in both fertilized and unfertilized hamster eggs showed no affinity for UEA-I and LTA, both of which recognize the external non-reducing fucose residues (Debray et al., 1981; Sugii and Kabat, 1982). However, fucose residues were detected in the zona pellucida of oocytes under all three experimental conditions when AAA was used as a probe. AAA, which preferentially binds $\alpha 1,6$-fucosyl residue linked to the core region of $N$-linked glycoproteins (Yamashita et al., 1985), showed an increase in the density of labelling in the inner portion of zona pellucida after fertilization. Previous in vitro fertilization and cytochemical studies demonstrated the role of fucose residues in sperm-zona pellucida recognition in guinea-pig, hamster, rat and human eggs (Huang et al., 1982; Shalgi et al., 1986; Mahony et al., 1991). In mice, Bleil and Wassarman (1988) reported that mouse zona pellucida treated with $\alpha$-L-fucosidase inhibits in vitro 
fertilization. The modifications of fucose residues detected by AAA after fertilization may be attributed to the release of specific enzymes or other glycoproteins into the perivitelline space of fertilized eggs. The alteration in the distribution of fucose residues in the inner layer of the zona pellucida of fertilized eggs probably reflects an alteration in the sperm binding sites, thus participating in the establishment of a block to polyspermy. However, more accurate tools are needed to support this hypothesis. In the present study, modifications of terminal Neu5Ac sugar residues, as recognized by MAA, were not detected after fertilization. Labelling by MAA is indicative of the occurrence of $\alpha(2,3)$ linked Neu5Ac in hamster zona pellucida glycoproteins; however, the presence of $\alpha(2,6)$-linked Neu5Ac was not detected with the lectin SNA. The other carbohydrate residue that may have a role as ZP3 sperm receptor is D-mannose. Pretreatment of human spermatozoa with D-mannose inhibited sperm penetration through the zona pellucida completely (Mori et al., 1989) and $\alpha$-methylmannoside and D-mannose are potent inhibitors of sperm penetration in rats (Shalgi et al., 1986) and mice (Cornwall et al., 1991). In the present study, the occurrence of $\alpha 1,3$ mannosyl residues in high mannose $\mathrm{N}$-linked oligosaccharides was not detected with GNA in fertilized, unfertilized and activated eggs.

In the present study, activation of cumulus-free eggs with the calcium ionophore A23187 was undertaken to obtain further insights into the anticipated post-activation modifications of various sugar residues in the zona pellucida. The cytochemical results of in vitro-activated eggs showed a different pattern of modifications with respect to the distribution of various lectin-binding sites over both the inner and outer portions of the zona pellucida compared with that of fertilized oocytes. Previous studies have demonstrated significant differences between parthenogenetic activation and in vivo fertilization (Raz et al., 1998). In addition, morphological studies in several species, including humans and hamsters, revealed the presence of two kinds of cortical granules with different electron densities (Ducibella et al., 1988; Okada et al., 1993). As such, Lens culnaris (LCA) agglutinin, a marker of cortical granule content, also showed two different types of binding after induction of cortical reaction by parthenogenetic activators (Raz et al., 1998). On the basis of the aforementioned studies, it is possible that the discrepancies in the density of labelling by the various lectins in the bilaminar zona pellucida between the fertilized and in vitro-activated eggs can be attributed to the differences in the composition of hydrolytic enzymes and glycoproteins contained in the two types of cortical granules, as well as in the timing of their discharge. In a dose-dependent study, Raz et al. (1998) demonstrated the occurrence of a full cortical reaction with the use of $2 \mu \mathrm{mol}$ calcium ionophore ionomycin $\mathrm{I}^{-1}$ as opposed to the partial cortical reaction resulting from the use of a lower concentration $\left(0.5 \mu \mathrm{mol} \mathrm{^{-1 }}\right)$ of the same artificial activator. Therefore, we are unable to rule out at this point that the concentration of calcium ionophore
A23187 used in the present study $\left(10 \mu \mathrm{mol} \mathrm{I}^{-1}\right)$ is a possible effector in bringing about a pattern of post-activation modifications, at least for the lectins used in the present study, that differ from those observed in both the inner and outer zona pellucida of fertilized oocytes. It is also possible that even a full cortical reaction induced parthenogenetically does not emulate the in vivo activation process by sperm penetration (Raz et al., 1998).

In conclusion, the results of the present study demonstrate that sugar residues contained in the zona pellucida glycoproteins of hamster eggs are modified after fertilization as well as after in vitro activation. They also provide further information about the asymmetric nature of the zona pellucida as demonstrated by quantitative analysis of lectingold labelling. The topographical distribution of various lectin-binding sites on the zona surface before and after fertilization indicates a possible role for different sugar residues in the process of sperm-zona pellucida binding, as acrosome-reacted spermatozoa are first observed at the outer surface of the zona pellucida. It has been argued that the initial sperm-egg interaction may involve components of low- and high-binding affinity, thereby indicating a hierarchy of binding events at the sperm-zona pellucida interface (Thaler and Cardullo, 1996), or possibly a different set of effectors including one or more sugar-recognizing proteins (Benoff, 1997). Modifications of sugar residues in the outer portion of the zona pellucida after fertilization, as is the case with WGA, Neu-WGA and RCA-I, may indicate a direct role of GlcNAc and galactose sugar residues, respectively, in the initial stages of recognition and binding between the spermatozoon and the zona pellucida. Of particular interest is the modification in the distribution of lectin-binding sites (HPA, RCA-I, WGA, Neu-WGA, BSAIB and $A A A)$ in the inner zona pellucida of fertilized eggs. It is likely that these modifications of the zona pellucida are required by the fertilized egg to prevent the lethal state of polyspermy from occurring. The detection of a high concentration of labelling with various lectins over the cortical granules of unfertilized eggs, as well as over the oolemma and perivitelline space of fertilized eggs, strongly supports the hypothesis for an important role of the cortical granules in contributing their contents of various enzymes towards the establishment of a block to polyspermy. Of particular importance are the differences in the pattern of lectin-gold labelling between the in vivo-fertilized and in vitroactivated eggs. Although the results obtained from our in vitro study confirm findings from previous studies regarding the role of cortical granule contents in modifying the biochemical structure of the zona pellucida after fertilization, it is possible that the results from our in vivo study represent a more accurate account of the distribution and modification of various sugar residues that might be involved in the various events of fertilization.

The authors wish to thank Hongmei Gu and John DaCosta for technical assistance, and Bob Temkin for reproduction of the original photomicrographs. This work was supported by a grant from the Canadian Institutes of Health Research (CIHR). 


\section{References}

Ahuja KK and Bolwell GP (1983) Probable asymmetry in the organization of components of the hamster zona pellucida Journal of Reproduction and Fertility 69 49-55

Avilés M, Martínez-Menárguez JA, Castells MT, Madrid JF and Ballesta J (1994) Cytochemical characterization of oligosaccharide side chains of the glycoproteins of rat zona pellucida: an ultrastructural study Anatomical Record 239 137-149

Avilés M, Jaber L, Castells MT, Kan FWK and Ballesta J (1996) Modifications of the lectin binding pattern in the zona pellucida of rat after fertilization Molecular Reproduction and Development 44 370-381

Avilés M, Jaber L, Castells MT, Ballesta J and Kan FWK (1997) Modifications of carbohydrate residue and ZP2 and ZP3 glycoproteins in the mouse zona pellucida after fertilization Biology of Reproduction $\mathbf{5 7}$ $1155-1163$

Avilés M, Castells MT, Abascal I, Martínez-Menárguez JA, Draber P, Kan FWK and Ballesta J (1999) Cytochemical localization of GalNAc and GalNAc $\beta 1,4 \mathrm{Gal} \beta 1,4$ disaccharide in mouse zona pellucida Cell and Tissue Research 295 269-277

Avilés M, El-Mestrah, Jaber L, Castell MT, Ballesta J and Kan FWK (2000a) Cytochemical demonstration of modification of carbohydrates in the mouse zona pellucida during folliculogenesis Histochemistry and Cell Biology 113 207-219

Avilés M, Okinaga T, Shur B and Ballesta J (2000b) Differential expression of glycoside residues in the mammalian zona pellucida Molecular Reproduction and Development 57 296-308

Benhamou N (1986) Preparation and application of lectin-gold complexes. In Colloidal Gold: Principles, Methods and Applications pp 95-139 Ed. MA Hayat. Academic Press, New York

Benoff S (1997) Carbohydrates and fertilization: an overview Molecular Human Reproduction 3 599-637

Berger T, Davis A, Wardrip NJ and Hedrick JL (1989) Sperm binding to the pig zona pellucida and inhibition of binding by solubilized components of the zona pellucida Journal of Reproduction and Fertility 86 559-565

Bleil JD and Wassarman PM (1988) Galactose at the non-reducing terminus of $O$-linked oligosaccharides of mouse egg zona pellucida glycoprotein ZP3 is essential for the glycoprotein's sperm receptor activity Proceedings National Academy of Sciences USA 85 6778-6782

Bleil JD, Beall CF and Wassarman PM (1981) Mammalian sperm-egg interaction: fertilization of mouse egg triggers modification of the major zona pellucida glycoproteins Developmental Biology 86 189-197

Bleil J, Greve J and Wassarman PM (1988) Identification of a secondary sperm receptor in the mouse zona pellucida: role in maintenance of binding of acrosome-reacted sperm to eggs Developmental Biology 128 376-385

Cahova M and Draber P (1992) Inhibition of fertilization by monoclonal antibody recognizing the oligosaccharide sequence GalNAc $\beta 1,4$ Gal $\beta 1,4$ on the mouse zona pellucida Journal of Reproductive Immunology 21 241-256

Cherr GN, Drobnis EZ and Katz DF (1988) Localization of cortical granule constituents before and after exocytosis in the hamster eggs Journal of Experimental Zoology 246 81-93

Cornwall GA, Tulsiani DRP and Orgebin-Crist MC (1991) Inhibition of the mouse sperm surface $\alpha$-D-mannosidase inhibits sperm-egg binding in vitro. Biology of Reproduction 44 913-921

Debray H, Decout D, Stecker G, Spik G and Montreuil J (1981) Specificity of twelve lectins towards oligosaccharides and glycopeptides related to $\mathrm{N}$-glycosylproteins European Journal of Biochemistry 117 41-55

Ducibella T (1991) Mammmalian egg cortical granules and the cortical reaction. In Elements of Mammalian Fertilization pp 205-231 Ed. PM Wassarman. CRC Press, Boca Raton

Ducibella TE, Anderson E, Albertini DF, Aalberg J and Rangarajan S (1988) Quantitative studies of changes in cortical granule number and distribution in the mouse oocyte during meiotic maturation Developmental Biology 130 184-197

Dunbar BS, Avery S, Lee V, Prasad SV, Schwahn D, Schwoebel, Skinner S and Wilkins B (1994) The mammalian zona pellucida: its biochemistry, immunochemistry, molecular biology and developmental expression Reproduction, Fertility and Development 6 59-76
El-Mestrah M and Kan FWK (2001) Distribution of lectin-binding glycosidic residues in hamster follicular oocytes and their modifications in the zona pellucida after ovulation Molecular Reproduction and Development $\mathbf{6 0}$ $517-534$

Fleming AD and Yanagimachi R (1980) Superovulation and superpregnancy in the golden hamster Development, Growth and Differentiation 22 103-112

Florman HM and Wassarman PM (1985) O-linked oligosaccharides of mouse egg ZP3 account for its sperm receptor activity Cell 41 313-324

Frens G (1973) Controlled nucleation for the regulation of the particle size in monodisperse gold suspensions Nature: Physical Science 241 20-22

Geoghegan WD and Ackerman GA (1977) Adsorption of horseradish peroxidase, ovomucoid and anti-immunoglobulin to colloidal gold for the indirect detection of concanavalin $\mathrm{A}$, wheat germ agglutinin and goat anti-human immunoglobulin $G$ on cell surfaces at the lectron microscopic level: a new method, theory and application Journal of Histochemistry and Cytochemistry 25 1187-1200

Gong X, Dubois DH, Miller DJ and Shur BD (1995) Activation of a G protein complex by aggregation of beta-1,4-galactosyltransferase on the surface of sperm Science $\mathbf{2 6 9} 1718-1721$

Hoodbhoy T and Tabot $\mathbf{P}$ (2001) Characterization, fate, and function of hamster cortical granule components Molecular Reproduction and Development 58 223-235

Huang TTF, Ohzu E and Yanagimachi R (1982) Evidence suggesting that L-fucose is part of a recognition signal for sperm-zona pellucida attachment in mammals Gamete Research 5 355-361

Johnston DS, Wright WW, Shaper JH, Hokke CH, Van den Eijinden DH and Joziasse DH (1998) Murine sperm-zona binding, a fucosyl residue is required for a high affinity sperm-binding ligand. A second site on sperm binds a non-fucosylated, $\beta$-galactosyl-capped oligosaccharide Journal of Biological Chemistry 278 1888-1895

Keefe D, Tran P, Pelligrini C and Oldenberg R (1997) Polarized light microscopy and digital image processing identify a multilaminar structure of the hamster zona pellucida Human Reproduction 2 $1250-1252$

Kimura J, Sato K, Tsukise A and Okano M (1991) Appearance of lectin binding affinity to the zona pellucida during hamster oocyte maturation Cellular and Molecular Biology 37 623-629

Litscher ES, Jununen K, Seppo A, Pentilla L, Niemela R, Renkonen $O$ and Wassarman PM (1995) Oligosaccharide constructs with defined structures that inhibit binding of mouse sperm to unfertilized eggs in vitro. Biochemistry 34 4662-4669

Mahony MC, Oehninger S, Clark GF, Acosta AA and Hodgen GD (1991) Fucoidin inhibits the zona pellucida-induced acrosome reaction in human spermatozoa Contraception 44 657-665

Martínez-Menárguez JA, Ballesta J, Avilés M, Castell MT and Madrid JF (1992) Cytochemical characterization of glycoproteins in the developing acrosome of rats. An ultrastructural study using lectin histochemistry, enzymes and chemical deglycosylation Histochemistry 97 439-449

Miller DJ, Macek MB and Shur BD (1992) Complementarity between sperm surface $\beta$-1,4-galactosyltransferase and egg-coat ZP3 mediates spermegg binding Nature 357 589-593

Miller DJ, Gong X, Decker G and Shur BD (1993) Egg cortical granule Nacetylglucosaminidase is required for the mouse zona block to polyspermy Journal of Cell Biology 123 1431-1440

Moller CC, Bleil JD, Kinloch RA and Wassarman PM (1990) Structural and functional relationship between mouse and hamster zona pellucida glycoproteins Developmental Biology 137 276-286

Neill JM and Olds-Clarke P (1988) Incubation of mouse sperm with lactate delays capacitation and hyperactivation and lowers fertilization levels in vitro. Gamete Research 20 459-473

Okada A, Inomata K and Nagae T (1993) Spontaneous cortical granule release and alteration of zona pellucida properties during and after meiotic maturation of mouse oocytes Anatomical Record 237 518-526

Phillips DM and Shalgi RM (1980) Surface properties of the zona pellucida Journal of Experimental Zoology 213 1-8

Piller V, Piller F and Cartron JP (1990) Comparison of the carbohydratebinding specificities of seven $\mathrm{N}$-acetyl-D-galactosamine-recognizing lectins European Journal of Biochemistry 191 461-466 
Prasad SV, Wilkins B, Skinner SM and Dunbar BS (1996) Evaluating zona pellucida structure and function using antibodies to rabbit $55 \mathrm{kDa}$ ZP protein expressed in baculovirus expression system Molecular Reproduction and Development 43 519-529

Rankin T, Talbot P, Lee E and Dean J (1999) Abnormal zonae pellucidae in mice lacking ZP1 result in early embryonic loss. Development 126 3847-3855

Raz T, Skuletsky E and Shalgi R (1996) Post-fertilization changes in the zona pellucida glycoproteins of rat eggs Histochemistry and Cell Biology 106 395-403

Raz T, Ben-Yosef D and Shalgi R (1998) Segregation of the pathways leading to cortical granule reaction and cell cycle activation in the rat egg Biology of Reproduction 58 94-102

Roth J (1983) Application of lectin-gold complexes for electron microscopic localization of glycoconjugates on thin sections Journal of Histochemistry and Cytochemistry 31 987-999

Shalgi RM, Matityahu A and Nebel L (1986) The role of carbohydrates in sperm-egg interaction in rats Biology of Reproduction 34 446-452

Shimuzu S and Ito M (1986) Glycoproteins of murine zona pellucida from unfertilized eggs and two-cell embryos: comparison of the reactivity to lectins Biochemistry International 13 335-342

Shur BD (1993) Glycosyltransferases as cell adhesion molecules Current Opinions in Cell Biology 5 854-863

Sugii S and Kabat EA (1982) Further immunochemical studies on the combining sites of Lotus tetragonolobus and Ulex europaeus I and II lectins Carbohydrate Research 99 99-101

Thaler CD and Cardullo RA (1996) The initial molecular interaction between mouse sperm and the zona pellucida is a complex binding event Journal of Biological Chemistry 27123 289-23 297
Tulsiani DRP, Yoshida-Komiya H and Araki Y (1997) Mammalian fertilization: a carbohydrate-mediated event Biology of Reproduction 57 487-494

Wassarman PM (1988a) The mammalian ovum. In: the Physiology of Reproduction pp 69-134 Eds E Knobil and JD Neill. Raven Press, New York

Wassarman PM (1988b) Zona pellucida glycoproteins Annual Review of Biochemistry 57 415-442

Wu AM and Sugii S (1991) Coding and classification of D-galactose, $N$-acetyl-D-galactosamine, and $\alpha$-D-Gal $p$-[1-3(4)]- $\beta$-D-Glc $p N A c$, specificities of applied lectins Carbohydrate Research 213 127-143

Yamashita K, Kochibe N, Ohkura T, Ueda I and Kobata A (1985) Fractionation of L-fucose-containing oligosaccharides on immobilized Aleuria aurantia lectin Journal of Biological Chemistry $2604688-4693$

Yanagimachi R and Nicolson GL (1976) Lectin-binding properties of hamster egg zona pellucida and plasma membrane during maturation and preimplantation Experimental Cell Research 100 249-257

Yonezawa N, Aoki H, Hatanaka Y and Nakano M (1995) Involvement of N linked carbohydrate chains of pig zona pellucida in sperm-egg binding European Journal of Biochemistry 233 35-41

Yurewicz EC, Pack BA and Sacco AG (1991) Isolation, composition and biological activity of sugar chains of porcine oocyte zona pellucida $55 \mathrm{~K}$ glycoproteins Molecular Reproduction and Development 30 126-134

Received 4 May 2001.

First decision 25 July 2001.

Revised manuscript received 13 December 2001.

Accepted 23 January 2002. 